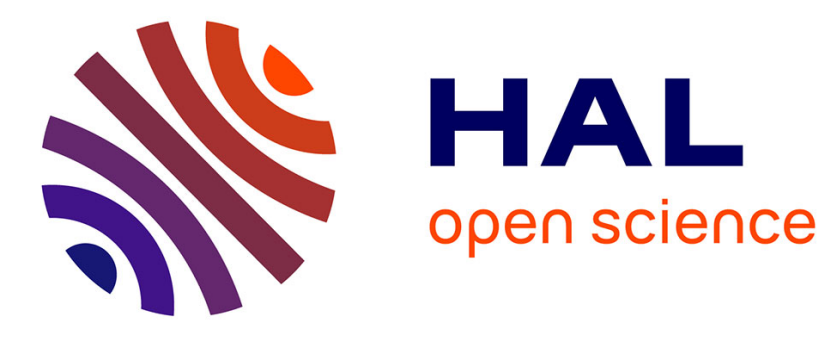

\title{
Job Shop Scheduling by pheromone approach in a dynamic environment \\ Paolo Renna
}

\section{To cite this version:}

Paolo Renna. Job Shop Scheduling by pheromone approach in a dynamic environment. International Journal of Computer Integrated Manufacturing, 2010, 23 (05), pp.412-424. 10.1080/09511921003642170 . hal-00588355

\section{HAL Id: hal-00588355 \\ https://hal.science/hal-00588355}

Submitted on 23 Apr 2011

HAL is a multi-disciplinary open access archive for the deposit and dissemination of scientific research documents, whether they are published or not. The documents may come from teaching and research institutions in France or abroad, or from public or private research centers.
L'archive ouverte pluridisciplinaire HAL, est destinée au dépôt et à la diffusion de documents scientifiques de niveau recherche, publiés ou non, émanant des établissements d'enseignement et de recherche français ou étrangers, des laboratoires publics ou privés. 


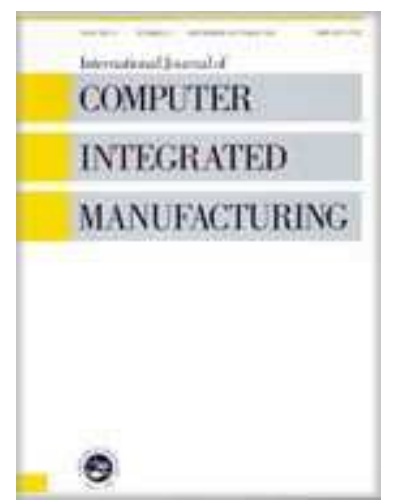

Job Shop Scheduling by pheromone approach in a dynamic environment

\begin{tabular}{|r|l|}
\hline Journal: & International Journal of Computer Integrated Manufacturing \\
\hline Manuscript ID: & TCIM-2009-IJCIM-0076.R1 \\
\hline Manuscript Type: & Original Manuscript \\
\hline $\begin{array}{r}\text { Date Submitted by the } \\
\text { Author: }\end{array}$ & $19-$ Nov-2009 \\
\hline Complete List of Authors: & Renna, Paolo; University of Basilicata, DIFA \\
\hline Keywords: & $\begin{array}{l}\text { DYNAMIC SCHEDULING, MULTI-AGENT SYSTEMS, DISCRETE EVENT } \\
\text { SIMULATION }\end{array}$ \\
\hline Keywords (user): & Ant Colony Intelligence, Pheromone \\
\hline
\end{tabular}

\section{$\diamond$ ScholarONE \\ Manuscript Central}




\title{
Job Shop Scheduling by pheromone approach in a dynamic environment
}

\begin{abstract}
Job shop scheduling problem is a NP-hard problem, therefore the objective is to create a schedule that satisfies all the constraints while taking as little overall time as possible. The paper concerns the job shop scheduling problem in cellular manufacturing systems; the schedule is created by a pheromone based approach. The proposed approach is carried out by a Multi Agent Architecture and it is compared with a coordination approach proposed in literature used as a benchmark.

A simulation environment developed in ARENA® package was used to implement the approaches and evaluate the performance measures. The performance measures investigated are: throughput time, throughput, Work In Process, machines average utilization and tardiness. Several scenarios are considered: from static to very dynamic conditions for internal and external exceptions of the manufacturing system. The simulation results highlighted that the performance of the proposed approach are comparable with the benchmark when the customer demand has a high fluctuation and the manufacturing system is less dynamic.
\end{abstract}

Keywords: Dynamic Scheduling, Ant Colony Intelligence, Pheromone, Multi Agent Systems, Discrete Event Simulation

\section{Introduction}

The environment within which manufacturing systems operate is characterized by rapid change than ever before. The unforeseen disturbances occur frequently and the manufacturing system has to be able to react to these disturbances. The disturbances can be: machine breakdowns, demand variability and delay in processing time. The above reasons drive the manufacturing systems to adopt dynamic scheduling approaches.

Dynamic scheduling has been defined under four categories (Mehta and Uzsoy, 1999; Vieira et al., 2000a, 2003; Aytug et al., 2005; Leus and Herroelen, 2005): 
- completely reactive scheduling; in this case no schedule in advance is created and the job schedule is obtained in a real-time fashion.

- predictive-reactive scheduling; a schedule is created in advance, but a rescheduling is considering to respond to exceptions in real-time.

- robust predictive-reactive scheduling; a schedule is created in advance and rescheduling activity is activated when the effect on the performance measures of the exceptions is significantly.

- robust pro-active scheduling; the schedule is computed in advance predicting the effect of exceptions on the manufacturing system.

The dynamic scheduling problem can be solved by using the following techniques:

- $\quad$ mathematical programming approach;

- $\quad$ dispatching rules approach;

- $\quad$ heuristic approach;

- $\quad$ artificial intelligence approach.

Moreover, most of the scheduling systems developed in industrial environments are centralized and hierarchical, but these approaches present several drawbacks. The most important drawbacks are the following:

- $\quad$ a central computer; it constitutes a bottleneck with a limit of capacity and a failure of it leads to bring down the entire manufacturing system.

- $\quad$ it is more difficult to extend and modify the configuration of the scheduling system;

- $\quad$ a slow response to disturbance because the information has to flow to the high level and then a reaction is planned.

Therefore, a centralized and hierarchical scheduling is inefficient in a very dynamic environment where the exceptions are more frequently.

For the above reasons, a decentralized control method is more efficient in a dynamic environment in particular, Multi Agent System methodology is more suitable for the implementation of decentralized system. A Multi Agent System (MAS) can be defined as a network of problem solvers 
that work together to solve problems that are beyond their individual capabilities (O'Hare and Jennings, 1996).

MAS approaches are more suitable to develop agile and robust distributed control, but its performance is more dependent by the coordination mechanism. The coordination mechanisms proposed in literature are the Contract net protocol (Smith 1980), market based, auction based (Siwamogsatham and Saygin, 2004) and game theory. These approaches have same limitations as: communication overhead, constantly exchange of information and therefore a minor reactivity of the agents.

Recently, many authors have developed several approaches inspired by the behavior of social insects like ants, bees, termites and wasp to propose an alternative method for coordination in complex systems. The most promising approach is based on Ant Colony; Ant Colony coordination refers to the cooperative ant foraging behavior. Ant colonies can always find shorter paths from a nest to a food source by pheromone trail laying and following. Dorigo et Al. (1991) first introduced Ant Colony Optimization (ACO) for solving the Traveling Salesman Problem, which is based on ant foraging. In manufacturing scheduling problem, the approach can be inspired to pheromonebased rule of Ant Colony to coordinate the MAS architecture.

The aim of this paper is to investigate the performance of a pheromone approach for cellular manufacturing systems in a very dynamic environment. The pheromone approaches proposed are two: one based on moving average and the other one on exponential moving average.

Two different types of disturbances are considered:

- internal exceptions; these exceptions are caused by the resources of the manufacturing system, in particular, they are: machine breakdowns and efficiency of the manufacturing machine.

- external exceptions; these exceptions are caused by external changes of the manufacturing system, specifically they are: changes in volume and mix demand products. 
Moreover, it is considered the effect of dispatching rule on the performance of the manufacturing system. The performance are compared with an approach proposed in literature based on classical coordination mechanism in MAS.

The structure of the paper is as follows: in Section 2 an overview of literature is presented. The manufacturing context is described in Section 3. In Section 4 the proposed coordination approaches based on pheromone are explained. The simulation environment and design of experiment conducted are given in Section 5, while in Section 6 the simulation results are presented. Finally, conclusions and future research path are presented in Section 7.

\section{Literature review}

Recently, a few researchers have developed approaches based on Ant Colony inspired scheduling in shop floor control. Most of the applications proposed concern the shop floor routing and permutation flow-shop sequencing problem

Peeters et al. (2001) has been developed a concept control system based on coordination mechanism of insect colonies, in particularly, a pheromone based control scheme is introduced. Basic principles of the pheromone concept, the control system architecture and a layered approach for decisionmaking have been discussed. Test beds of industrial scale have been used to demonstrate properties and benefits of this approach. The main advantages emerged for the pheromone approaches are: a simple coordination mechanism; the automatic guidance to the optimized solution; the capability to handle dynamic situations.

Yu and Ram (2006) proposed Multi Agent approach designed for dynamic job shops with routing flexibility and sequence-dependent setup. A bio-inspired strategy based on division of labour in insect societies is presented for coordination among agents. The strategy is accomplished using a computational model, which is composed of response threshold, response intention, and machinecentred reinforcement learning. The bio-inspired scheduling is compared with an agent-based approach and a dispatching rule-based approach. The experiments were performed using simulation 
and statistical analysis. Results show that the proposed bio-inspired scheduling model performs better than the other two methods on all eight common scheduling metrics.

Xiang and Lee (2008) presented research concerning dynamic scheduling by agent coordination that is inspired on Ant Colony in MAS. The proposed approach is tested in a shop floor model considers multiple job types and parallel multi-purpose machines with dependent setup times. Moreover, the machine break down is included as a disturbance of the manufacturing system. In this research, the Ant Colony is used to find an appropriate machine agent for processing and helps the machine agent to determine the next job to be processed in the current queue.

Scholz-Reiter et al. (2008) proposed a pheromone-based autonomous control method to a mix model of a shop floor and tested it in different dynamic demand situations. They compared the pheromone approach with a method based on queue length estimator. The only performance is the throughput time with a sinusoidal arrival rate of the parts.

Zhou et al. (2009) proposed an algorithm based on Ant Colony Optimization in a shop floor scenario with three levels of machine utilization, three different processing time distributions, and three different performance measures for intermediate scheduling problems. The performances measured are: mean flow time, mean tardiness, total throughput on different experimental environments are compared with those from dispatching rules including first-in-first-out, shortest processing time, and minimum slack time. The experimental results show that ACO outperforms other approaches when the machine utilization or the variation of processing times is not high. The procedure proposed is a centralized approach.

Renna (2009) developed two pheromone approaches for the job shop scheduling problem. One is based on the past information of a part (throughput time of the manufacturing cell); and the other on the queue of the manufacturing cell. The proposed approaches are tested in dynamic environment; the simulation results show how the approach based on the queue of the manufacturing cell performs better when the environment conditions are very dynamic.

Based on the above literature review, the following limitations can be drawn: 
- a benchmark based on MAS architecture with complex coordination mechanism wasn't used in order to evaluate the advantages and disadvantages of Ant Colony coordination mechanisms;

- the proposed approaches in literature are tested in manufacturing systems in which some exceptions occur and the analysis on the rapidity of alterations was not investigated.

- each model proposed is tested on few performance measures (for example only throughput performance);

The main findings of the research proposed in this paper can be summarized as it follows:

- the pheromone approach is deeply investigated by an adequate number of performance measures;

- the simulations have been conducted in a several dynamicity levels introducing both internal and external disturbances;

- the pheromone approaches have been compared with a complex MAS approach in order to find out in which environment conditions the proposed approach can be competitive.

\section{Manufacturing system context}

The manufacturing system consists of a given number of cells; each cell is able to perform any kind of manufacturing operation so that the resulting manufacturing system is a pure general-purpose one. In such a system, the scheduling decision consists in deciding in what manufacturing cell the part will perform the next operation, therefore a pure dispatching problem. The manufacturing system has the following characteristics:

- several part types have to be manufactured by the manufacturing system. Each part type has a predefined number of operations performed by the manufacturing cells. At each part is assigned a due date.

- orders for production of different parts arrive randomly with an inter-arrival that is exponential distribution; 
- each machine performs the manufacturing operation with an efficiency, which sets the speed of the operation.

- the queues are managed by the First In First Out policy in order to investigate only the pheromone approaches policy.

- each machine can breakdown randomly with an exponential distribution.

In this research, the transportation time of the material handling devices are included in the processing time, and the handling resources are always available.

The coordination approach based on Ant Colony is inspired by the behavior of foraging ants that leave a pheromone trail on their way to the food. In the real world, ants (initially) wander randomly, and upon finding food return to their colony while laying down pheromone trails. If other ants find such a path, they are likely not to keep traveling at random, but instead follow the trail, returning and reinforcing it if they eventually find food. Over time, however, the pheromone trail starts to evaporate, thus reducing its attractive strength.

Ant Colony is a natural example of highly distributed system, therefore, it can be formalized by Multi Agent System. The coordination mechanism based on Ant Colony has the following advantages:

- The simplicity of coordination mechanism. In fact, the ants do not communicate directly each other, but the communication is performed by the pheromone released on the environment. The ants have to know how to put information and how to get information from the environment.

- The evaporation of the pheromone is the methodology how this coordination approach is capable to handle dynamic situations.

The drawbacks of pheromone approaches are the following:

- The delay of the information; the ants release a pheromone based on its activity; the other ants put this information with a delay time.

- The evaporation rate is a parameter to set, therefore an opportune study on the specific problem modeled has to be conduct. 
In a manufacturing system the ants are the parts that flow through the manufacturing cells; the manufacturing cells are the nodes that ant must visit. When a part leaves a manufacturing cell, it deposits a pheromone based on the throughput time in the manufacturing cell.

Therefore, the problems are mainly two:

- how the pheromone information are formalized, what type of information can be included;

- the methodology of pheromone evaporation.

The activities of Ant Colony algorithm for scheduling in manufacturing systems are the following:

- Initializing pheromone for each manufacturing cell;

- Update pheromone for each manufacturing cell;

- Pheromone evaporation.

\section{Pheromone approach}

In this paper, two pheromone formulations have been proposed: the first is based on moving average of the information deposited by the parts; the second is based on exponential moving average. The two formulations are an adaptation of the approaches proposed, respectively in Scholz-Reiter (2008) and Renna (2009). The main difference between the two approaches is the data stored; in moving average the amount of data for the computation is higher than the exponential moving average.

The information deposited by the parts for the pheromone computation is the throughput time of a part (ant) that is processed on a manufacturing cell (node).

In particular, the value deposited by a part is computed by the following expression:

value $=\frac{1}{\text { tnow }-\operatorname{tin}}$

where,

tnow is the time when the part leaves the manufacturing cell;

tin is the arrival time in the manufacturing cell of the part. 
From the above expression, the difference (tnow-tin) concerns the throughput time of the part in the manufacturing cell. Therefore, if the throughput time is low, then the pheromone deposited by the part is high.

The value of pheromone at start time of the simulation is obtained by the following expression:

value $=\frac{1}{\text { proces sing time }}$

where,

processing time is the value of processing time performed by the manufacturing cell.

\subsection{Pheromone formulation I}

The evaporation strategy of this approach is obtained by the computation of moving average of the value computed in expression 2. The pheromone is computed by moving average over last $N$ value deposited by the parts (expression 2). The older values deposited are not included, then, this emulates the evaporation of the pheromone.

$$
\text { pheromone }_{j}(t)=\frac{1}{N} \sum_{i=1}^{N} \text { value }_{i}
$$

Therefore, according to above description, the activities are the following:

- $\quad$ initialize pheromone (expression 2);

- update pheromone (expression 1);

- pheromone evaporation (expression 3).

The probability to select a manufacturing cell by the part agent, is in direct ratio to the value of pheromone $_{j}(t)$; specifically, the probability to select a manufacturing cell $j$ is

$\frac{\text { pheromone }_{j}(t)}{\sum_{j} \text { pheromone }_{j}(t)}$, with $j=1, \ldots .$, number of manufacturing cells.

This formulation of pheromone is a look back methodology, because the pheromone value is computed by past events. For example, if a machine is in breakdown status, the pheromone is reduced with a delay time that the other parts can "sniff". 


\subsection{Pheromone formulation II}

The difference from the first formulation is the evaporation strategy based on exponential moving average. The pheromone is computed by the following expression:

$$
\text { pheromone }_{j}(t)=(1-\alpha) \bullet \text { pheromone }_{j}(t-1)+\alpha \bullet \text { value }^{\prime}
$$

The above expression is the average between the last value memorized (pheromone $j(t-1))$ and the value computed by the part that leaves the manufacturing cell (value).

The value $\alpha$ (it is in range [0,1]) emulates the evaporation of the pheromone. In particular, a high value of $\alpha$ leads to high evaporation because the value is more significant and vice versa for low value of $\alpha$. Therefore, according to above description, the activities are the following:

- initialize pheromone (expression 2);

- update pheromone (expression 1);

- evaporation pheromone (expression 4).

\subsection{The efficiency based approach (benchmark Renna et al. (2001))}

Briefly, in this approach, the MAS is composed by two types of agent: part agent and manufacturing cell agent. The productive function of the manufacturing cell agent consists in evaluating and providing to the part agent the following three parameters: Expected Part Throughput Time (ETT); Resource Failure Index (RFI); Resource Processing Time Index (RPTI). The ETT is computed by the following expression:

StdFlowTime $_{k}(t)=\frac{\text { medwtime }}{\text { medwtime }+ \text { FlowTime }}(t)$

medwtime is the expected processing time of the parts.

FlowTime $_{k}(t)$ is the expected manufacturing cell $k$-th throughput time computed by summing up the processing times of the parts waiting in the manufacturing cell queue plus the residual service time of the part being worked in the machine at the negotiation time $t$. This index is the measure of workload of the generic manufacturing cell. The index value is one if no parts are in queue and the 
resource is in idle state; it decreases with the increase of parts in queue. The RFI is computed by the following expression

$R F I_{k}(t)=1-\frac{F T_{k}(t)}{t}$

where, $F T_{k}(t)$ is the total time of failure status of the resource $k$ until the negotiation time $t$.

The $R P T$ is the processing time in the generic manufacturing cell, it is obtained by the $e f f_{k}(t)$ (see table 2) multiplied to medwtime.

Each parameter is normalized for each $k$-th resource, by the following expression:

$$
\text { Normalized Index } x_{k}(t)=\frac{\max _{k} \operatorname{index}(t)-\operatorname{index}(t)}{\max _{k} \operatorname{index}(t)-\min _{k} \operatorname{index}(t)}
$$

Then, each index is normalized between the maximum and minimum values of the indexes among all the manufacturing cells.

The above indexes are composed by the following expression:

Eff Index $=w \cdot$ NormEPPT $+(1-w) \cdot($ NormRFI + NormRPT $)$

The part agent selects the manufacturing cell that has the high value of efficiency. In this paper the value of weight $w=0.4$ that leads to better performance.

The above approach needs to implement a Multi Agent infrastructure with an exchange of information bi-directional and with a computation of this information. Therefore, the approach is more complex in terms of infrastructure and computational time.

\section{Simulation environment}

In order to evaluate the performance of the proposed approaches a simulation environment is developed. The author selected the Arena® discrete event simulation platform by Rockwell Software Inc. it was used to develop the simulation model of the presented approaches. The manufacturing system consists of four general-purpose manufacturing cells that are called to manufacture a set of four different parts. In order to emulate a dynamic environment the proposed approaches have been tested through a production run consisting of several alternating stages; each 
stage is characterized by different internal (efficiency of the manufacturing cells, and machine breakdowns) and external attributes (mix and inter-arrival demand).

The processing time of a manufacturing cell is characterized by an efficiency parameter (eff); in particular, the processing time is obtained by the following expression:

processing time $=e^{e f f} \cdot e P T$

where, $e P T$ is the expected Processing Time (medwtime) for all the parts equal to 30 minutes.

\section{[Insert table 1 about here]}

Table 1 reports the experimental classes tested; the parameters concerning:

- Manufacturing operations; it is the number of manufacturing operations that the parts need. The classes from one to twenty four are characterized by the number of operations equal for all the parts, while from twenty five to thirty nine classes the manufacturing operations are different for the parts and the values are reported in table 3.

- Mix; it is the mix of the four typology parts reported in table 4.

- Internal disturbance; it is the static or dynamic change of machine breakdowns and efficiency parameters. The values are reported in table 2.

- Inter-arrival time dynamic; it is the dynamic change of the inter-arrival time of the parts (see table 5).

- Congestion level; it is the level of workload of the manufacturing system. In case of dynamic inter-arrival time the congestion level is variable over the simulation length.

- Dynamicity level; it is the rapidity changing of dynamic conditions (see table 6).

The evaporation parameter of the pheromone approach I is carried out by the computation of the last five value deposited. The simulations conducted for different values of $N$ (see equation 3 ) highlight that five is the number of $N$ that leads to better results.

The evaporation parameter of the pheromone approach II $\alpha$ is fixed to 0.5 in order to evaluate with the same importance between the last value stored and the new pheromone deposited by the part. 
It has been assumed that all the manufacturing cells are subject to faults, and failures occur in accordance with exponentially distributed time between failures, with mean time between failure $(M T B F)$. Repairing times are also exponentially distributed with mean time to repair (MTTR).

Table 2 reports the parameter of the exponential distribution of MTBF and Efficiency (Eff) for each manufacturing cell and each stage. As the reader can notice, the MTBF and Eff decrease the performance of the manufacturing cell in order to emulate a degradation of the machines.

The MTTR is related to the processing time by the following expression:

Mean Time To Repair $=1.5 * e P T$.

\section{[Insert table 2 about here]}

Table 3 reports the number of visit of the four typology parts in order to investigate the performance over the number of visit and for different number of visit for the typology parts.

\section{[Insert table 3 about here]}

Table 4 reports the mix changes over the stages of the simulation.

\section{[Insert table 4 about here]}

Parts enter the system following an exponential arrival stream; the inter-arrival times (exponential parameter) are computed for the different experimental classes in order to keep the three levels of workload (congestion) reported in table 1. The workload index allows to keep the same congestion level (high, medium and low) when the other experimental conditions change; it is computed as follows:

workload index $=\frac{\text { int er }- \text { arrival }{ }^{*} 4}{\text { number of visit }{ }^{*} 30}$

where,

inter-arrival is the parameter of the exponential arrival stream (excepted value); number of visit is reported in table 3 ;

4 is the number of manufacturing cell;

30 is the expected processing time. 
Then, the inter-arrival time parameter is computed in order to obtain a value of expression 10 respectively:

- $\quad$ workload index=1.2 for low congestion level;

- $\quad$ workload index=1 for medium congestion level;

- $\quad$ workload index $=0.8$ for high congestion level.

Finally, the dynamic change of the inter-arrival time is reported in table 5, in which low and high fluctuation of the inter-arrival parameter are considered. To model a highly dynamic demand, the inter-arrival time is oscillating between two values with situation of over and under load. Table 5 reports two levels of fluctuation of the inter-arrival time.

\section{[Insert table 5 about here]}

Moreover, specific due date has been assigned to each part entering the system. Denoting with $t_{j}^{\text {now }}$ the arrival time of a specific part $j$, its due date follows an uniform distribution in the range has given in equation (11). The objective is to investigated the difference among the proposed approaches and benchmark, therefore the range in equation (11) has minor importance.

If the part leaves the system after this due date, a penalty is computed equal to such a delay (tardiness).

$d d_{j}=t_{j}^{\text {now }}+U N I F O R M[3,8] \bullet e P T \bullet O p N u m b$

where $O p N u m b$ is the number of visit of the specific part $j$.

The proposed approaches are tested in static and highly dynamic situations; the dynamicity of the manufacturing system is characterized by the stage length factor. The simulation length is fixed to 14400 time units in order to avoid transitory influence. During the stage length the characteristics of the manufacturing system are constant, therefore the rapid succession of stages control the dynamicity of the manufacturing system conditions. The length of the stage is related to the expected processing time by the stage length factor :

stage lenght $=$ stage lenght factor $\bullet$ ePT 
For each experimental class ( expect for static classes) the simulations are conducted for three levels of dynamicity as reported in table 6. The level low leads to repeat each stage once, level medium leads to repeat each stage twice and level high leads to repeat each stage three times.

\section{[Insert table 6 about here]}

Furthermore, because of the parameters extracted by the exponential distribution (inter-arrival time, machine breakdown), and in order to guarantee a statistical validity of the results, for each run, the number of executed replications guarantees, for the output performance measures, that the length of confidence intervals (95\% level) of the mean among replications is lower than $5 \%$ of the mean itself. The performance measures investigated are: throughput time, throughput, Work In Process, machines average utilization and tardiness.

\section{Simulation Results}

The simulation results have been reported in the following figures. The Figures report the percentage difference of the proposed pheromone approaches compared to efficiency approach (base approach for determining percentage difference).

\section{[Insert figure 1 about here]}

Figure 1 reports the average flow time in static and dynamic conditions. The proposed approaches lead to best performance when the system is in high utilization state with internal exceptions (Exp. No. 10,11 and 12 see table 1), and the exponential approach is always the best approach.

Concerning the throughput, the difference among the approaches is always under one percent. Therefore, the three approaches (efficiency and the two pheromone formulations) have the same level of throughput. The Work In Process measure has the same trend of the flow time performance. The average utilization of the manufacturing system is the same among the different approaches.

[Insert figure 2 about here] 
Figure 2 reports the tardiness performance. The better performance is obtained when the system is in high congestion level with internal exceptions, however the pheromone approaches lead to a great reduction of this performance.

All the performance measures get worse when the dynamicity increases and the congestion level decreases. Therefore, the proposed approaches are suitable to be used in condition of low or medium dynamicity of internal exceptions and when the congestion level is high. The exponential moving average is better than the moving average.

The above simulation results have been conducted in a manufacturing system where the parts require only one manufacturing operation. Then, the approaches proposed are tested when the manufacturing operations increasing and they are different for each typology part.

\section{[Insert figure 3 about here]}

Figure 3 shows that the flow time is the same for different number of operations and when it is introduced the mix dynamicity among the typology parts. Except the case with three manufacturing operations for all the typology parts, in this case the performance of the proposed approaches are better than the other cases (Exp. No. 20 and 21). However, the number of manufacturing operations has a low effect on the performance. The Work In Process performance has the same trend.

\section{[Insert figure 4 about here]}

The throughput performance decreases in a manufacturing system with three or four operations for all the part typology, while this performance is the same of the efficiency approach in case of manufacturing operations different for the typology part and with mix dynamicity (as showed in figure 4). Then the number of operations leads to reduce the throughput of the manufacturing system that adopts the pheromone formulations.

\section{[Insert figure 5 about here]}

Figure 5 shows that the different manufacturing operations for the product typology and the mix dynamicity lead to worst tardiness measure. Moreover, the performance get worse when the 
dynamicity is high. This confirms that the pheromone approaches are worst when external exceptions occur.

A further exception is introduced; in particular a fluctuation of the inter-arrival time between two values that lead to over and under loaded of the manufacturing system. Two levels are considered: a low range between the two values of inter-arrival; a high range between the two values of interarrival.

\section{[Insert figure 6 about here]}

From the figure 6 two issues can be drawn:

- If the oscillation of the inter-arrival time is very wide, the performance of the pheromone approaches are competitive with the efficiency approach;

- The advantage of the efficiency approach increases with the increase of the dynamicity of the system.

Therefore, the pheromone approaches improve its performance when the external exceptions are considerable. The Work In Process performance has the same trend.

\section{[Insert figure 7 about here]}

In todays competitive environment, a high level of delivery performance has become very important. For this reason, it is analyzed how the earliest due date (EDD) rule can improve the performance of the proposed approaches. Then, the queues of the manufacturing cells are managed by the EDD rule. Figure 8 shows that the pheromone approach decreases the performance index with the EDD rule. The moving average has a tremendous decrement of the performance, while the exponential moving average reduction is limited. The same trend is verified for the tardiness performance. Therefore, the EDD rule leads to reduction all the performance measures.

\section{[Insert figure 8 about here]}

The same trend is verified for the tardiness performance (see figure 9). Therefore, the EDD rule leads to reduction all the performance measures and the due date performance too (tardiness).

\section{[Insert figure 9 about here]}




\section{Conclusions and future development}

The research presented a deeply discussion on dynamic scheduling by pheromone approach. This approach is inspired by Ant Colony Intelligence; the pheromone approach is simpler than traditional approaches based on MAS coordination (contract net, market like, etc.), but the performance have to be investigated. A simulation environment has been developed to test the proposed approaches in a generic manufacturing system. The simulations have been conducted is several conditions and in a very dynamic environment with internal (machine breakdowns and machine efficiency) and external (mix and inter-arrival time) exceptions. The pheromone formulation is based on two approaches: moving average and exponential moving average. Moreover, a benchmark approach is used to evaluate the performance measures.

The results of this research can be summarized as it follows:

- The throughput and the average utilization of the manufacturing system are the performance measures that have the same values of the benchmark.

- The pheromone approach is competitive when internal and external exceptions occur and the fluctuation of the inter-arrival demand is high.

- The pheromone approach based on exponential moving average outperforms the moving average; therefore the exponential moving average reacts faster to the manufacturing system exceptions. This is important, because the exponential moving average is simpler than the moving average for the data management.

- A significant result is how the pheromone approaches react to the dispatching rules. In this research is introduced the EDD rule; the simulation results show that the moving average leads to reduce dramatically the performance, while for the exponential moving average the reduction is minor. Therefore, the dispatching rules have to be integrated in the pheromone computation. 
The reduction of the performance when the dynamicity increases is caused by the delay information of pheromone approach; when the dynamicity is higher than the stage length the pheromone approach is unable to compete with efficiency approach.

A further research paths can be the following:

The influence of the manufacturing system size on the performance measure. The study of the performance measures when a high number of manufacturing cells characterizes the manufacturing system. The increase of the manufacturing cells lead to a more complex scheduling problem; therefore a deeply investigation of the pheromone approach responsiveness to a complexity of scheduling problem has been performed.

The extension of the research to the material handling system and the development of the pheromone approach for both scheduling and routing of the parts.

- $\quad$ The development of hybrid approach; in this case, the dynamic scheduling approach can be adapted to the environment conditions. Therefore, when the dynamicity is high, complex method is activated, otherwise the pheromone approach can be performed. This allows reducing the computational time and complexity to dynamic scheduling in manufacturing systems.

\section{References}

Aytug, H., Lawley, M. A., McKay, K., Mohan, S., and Uzsoy, R., 2005. Execting production schedules in the face of uncertainties: A review and some future directions. European Journal of Operational Research, 161 (1), 86-110.

Dorigo, M., Maniesco, V., Colorni, A., 1991. Distributed optimization by ant colonies. Proceedings of the ECAL91 European Conference on Artificial Life, 134-142. 
Leus, R. and Herroelen, W., 2005. The complexity of machine scheduling for stability with a single disrupted job. Operations Research Letters, 33 (2), 151-156.

Mehta, S. V. and Uzsoy, R., 1999. Predictable scheduling of a single machine subject to breakdowns. International Journal of Computer Integrated Manufacturing, 12 (1), 15-38.

O'Hare, G. M. P. and Jennings, N. R. (eds.), 1996. Foundations of Distributed Artificial Intelligence. John Wiley \& Sons.

Peeters, P., Van Brussel, H., Valckenaers, P., Wyns, J., Bongaerts, L., Kollingbaum, M., Heikkila, T., 2001. Pheromone based emergent shop floor control system for flexible flow shops. Artificial Intelligence in Engineering, 15, 343-352.

Renna, P., 2009. A Performance Comparison between Efficiency and Pheromone Approaches in Dynamic Manufacturing Scheduling. Chapter 12 in Intelligent Systems for Automated Learning and Adaptation, edited by Raymond Chiong, Information Science Reference, Hershey, New York, pp. 273-298.

Renna, P., Perrone, G., Amico, M., Bruccoleri, M., Noto La Diega, S., 2001. A performance comparison between market like and efficiency based approaches in Agent Based Manufacturing environment. In 34th International Seminar for Manufacturing Systems, May 2001, Athens (Greece), 93-98.

Siwamogsatham, V. and Saygin, C., 2004. Auction-based distributed scheduling and control scheme for flexible manufacturing systems. International Journal of Production Research, 42, 547572. 
Smith, R.G., 1980. The Contract Net Protocol: high level communication and control in a distributed problem solver. IEEE Transactions on Computers C-29 (12), 1104-1113.

Scholz-Reiter, B., De Beer, C., Freitag, M., Jagalski, T., 2008. Bio-inspired and pheromone-based shop floor control. International Journal of Computer Integrated Manufacturing, 21 (2), 201-205.

Vieira, G. E., Herrmann, J. W., and Lin, E., 2000. Analytical models to predict the performance of a single machine system under periodic and event-driven rescheduling strategies. International Journal of Production Research, 38 (8), 1899- 1915.

Vieira, G. E., Hermann, J. W., and Lin, E., 2003. Rescheduling manufacturing systems: a framework of strategies, policies and methods, Journal of Scheduling, 6 (1), 36-92.

Xiang, W., Lee, H.P., 2008. Ant colony intelligence in multi-agent dynamic manufacturing scheduling. Engineering Applications of Artificial Intelligence 21,73-85.

Yu, X., Ram, B., 2006. Bio-inspired scheduling for dynamic job shops with flexible routing and sequence dependent setups. International Journal of Production Research, 44 (22), 4793-4813.

Zhou, R., Nee, A.Y.C. and Lee, H.P., 2009. Performance of an ant colony optimisation algorithm in dynamic job shop scheduling problems. International Journal of Production Research, 47(11), 2903-2920. 


\section{List of tables}

- Table 1. Experimental classes

- Table 2. Mean Time Between Failure (MTBF) and Efficiency (Eff)

- Table 3. Number of visit

- Table 4. Mix changes

- Table 5. Inter-arrival time chages

- Table 6. Experiment classes 


\begin{tabular}{|c|c|c|c|c|c|c|}
\hline $\begin{array}{c}\text { Exp. } \\
\text { No. }\end{array}$ & $\begin{array}{c}\text { Manufacturing } \\
\text { Operations }\end{array}$ & Mix & $\begin{array}{c}\text { Internal } \\
\text { disturbances }\end{array}$ & $\begin{array}{l}\text { Inter- arrival } \\
\text { time dynamic }\end{array}$ & $\begin{array}{c}\text { Congestion } \\
\text { Level }\end{array}$ & $\begin{array}{c}\text { Dynamicity } \\
\text { level }\end{array}$ \\
\hline 1 & One for all parts & Equal for all parts & Static & No & Low & - \\
\hline 2 & One for all parts & Equal for all parts & Static & No & Medium & - \\
\hline 3 & One for all parts & Equal for all parts & Static & No & High & - \\
\hline 4 & One for all parts & Equal for all parts & Dynamic & No & Low & Low \\
\hline 5 & One for all parts & Equal for all parts & Dynamic & No & Low & Medium \\
\hline 6 & One for all parts & Equal for all parts & Dynamic & No & Low & High \\
\hline 7 & One for all parts & Equal for all parts & Dynamic & No & Medium & Low \\
\hline 8 & One for all parts & Equal for all parts & Dynamic & No & Medium & Medium \\
\hline 9 & One for all parts & Equal for all parts & Dynamic & No & Medium & High \\
\hline 10 & One for all parts & Equal for all parts & Dynamic & No & High & Low \\
\hline 11 & One for all parts & Equal for all parts & Dynamic & No & High & Medium \\
\hline 12 & One for all parts & Equal for all parts & Dynamic & No & High & High \\
\hline 13 & One for all parts & Equal for all parts & Dynamic & Yes & Variable & Low \\
\hline 14 & One for all parts & Equal for all parts & Dynamic & Yes & Variable & Medium \\
\hline 15 & One for all parts & Equal for all parts & Dynamic & Yes & Variable & High \\
\hline 16 & Two for all parts & Equal for all parts & Dynamic & Yes & variable & Low \\
\hline 17 & Two for all parts & Equal for all parts & Dynamic & Yes & variable & Medium \\
\hline 18 & Two for all parts & Equal for all parts & Dynamic & Yes & variable & High \\
\hline 19 & Three for all parts & Equal for all parts & Dynamic & Yes & variable & Low \\
\hline 20 & Three for all parts & Equal for all parts & Dynamic & Yes & variable & Medium \\
\hline 21 & Three for all parts & Equal for all parts & Dynamic & Yes & variable & High \\
\hline 22 & Four for all parts & Equal for all parts & Dynamic & Yes & variable & Low \\
\hline 23 & Four for all parts & Equal for all parts & Dynamic & Yes & variable & Medium \\
\hline 24 & Four for all parts & Equal for all parts & Dynamic & Yes & variable & High \\
\hline 25 & Different & Equal for all parts & Dynamic & Yes & variable & Low \\
\hline 26 & Different & Equal for all parts & Dynamic & Yes & variable & Medium \\
\hline 27 & Different & Equal for all parts & Dynamic & Yes & variable & High \\
\hline 28 & Different & Dynamic changes & Dynamic & Yes & variable & Low \\
\hline 29 & Different & Dynamic changes & Dynamic & Yes & variable & Medium \\
\hline 30 & Different & Dynamic changes & Dynamic & Yes & variable & High \\
\hline 31 & Different & Dynamic changes & Dynamic & Low fluctuation & variable & Low \\
\hline 32 & Different & Dynamic changes & Dynamic & Low fluctuation & variable & Medium \\
\hline 33 & Different & Dynamic changes & Dynamic & Low fluctuation & variable & High \\
\hline 34 & Different & Dynamic changes & Dynamic & High fluctuation & variable & Low \\
\hline 35 & Different & Dynamic changes & Dynamic & High fluctuation & Variable & Medium \\
\hline 36 & Different & Dynamic changes & Dynamic & High fluctuation & variable & High \\
\hline 37 & Different & Dynamic changes & Dynamic & Low fluctuation & With EDD & Low \\
\hline 38 & Different & Dynamic changes & Dynamic & Low fluctuation & With EDD & Medium \\
\hline 39 & Different & Dynamic changes & Dynamic & Low fluctuation & With EDD & High \\
\hline
\end{tabular}

Table 1. Experimental classes 


\begin{tabular}{cccccc}
\hline & Stage 1 & Stage 2 & Stage 3 & Stage 4 & Stage 5 \\
\hline MTBF (1) [minutes] & 1000 & 800 & 640 & 512 & 409.6 \\
MTBF (2) [minutes] & 800 & 640 & 384 & 307.2 & 245.76 \\
MTBF (3) [minutes] & 600 & 480 & 256 & 204.8 & 163.84 \\
MTBF (4) [minutes] & 400 & 320 & 128 & 102.4 & 81.92 \\
Eff (1) [minutes] & 0.5 & 0.6 & 0.7 & 0.8 & 0.9 \\
Eff (2) [minutes] & 0.6 & 0.65 & 0.7 & 0.75 & 0.8 \\
Eff (3) [minutes] & 0.7 & 0.72 & 0.74 & 0.76 & 0.78 \\
Eff (4) [minutes] & 0.8 & 0.81 & 0.82 & 0.83 & 0.84 \\
\hline
\end{tabular}

Table 2. Mean Time Between Failure (MTBF) and Efficiency (Eff) 


\begin{tabular}{ccccc}
\hline Number of visit (table 1) & Part 1 & Part 2 & Part 3 & Part 4 \\
\hline From 1 to 6 Exp. No & 1 & 1 & 1 & 1 \\
From 7 to 9 Exp. No & 2 & 2 & 2 & 2 \\
From 10 to 12 Exp. No & 3 & 3 & 3 & 3 \\
From 13 to 15 Exp. No & 4 & 4 & 4 & 4 \\
\hline From 16 to 24 Exp. No & 4 & 3 & 2 & 1 \\
\hline
\end{tabular}

Table 3. Number of visit 


\begin{tabular}{cccccc}
\hline & Stage 1 & Stage 2 & Stage 3 & Stage 4 & Stage 5 \\
\hline Mix part 1 & 0.25 & 0.4 & 0.1 & 0.2 & 0.3 \\
Mix part 2 & 0.25 & 0.3 & 0.4 & 0.1 & 0.2 \\
Mix part 3 & 0.25 & 0.2 & 0.3 & 0.4 & 0.1 \\
\hline Mix part 4 & 0.25 & 0.1 & 0.2 & 0.3 & 0.4 \\
\hline
\end{tabular}

Table 4. Mix changes 


\begin{tabular}{cccccc}
\hline & Stage 1 & Stage 2 & Stage 3 & Stage 4 & Stage 5 \\
\hline Dynamic (workload index) & 0.8 & 1 & 1.2 & 0.8 & 1 \\
Low fluctuation [minutes] & 40 & 10 & 40 & 10 & 40 \\
\hline High fluctuation [minutes] & 50 & 10 & 50 & 10 & 50 \\
\hline
\end{tabular}

Table 5. Inter-arrival time chages 


\begin{tabular}{cccc}
\hline Dynamicity level & Stage length factor & Simulation run length & Stage repeat \\
\hline Low & 96 & 12400 & 1 \\
Medium & 48 & 12400 & 2 \\
\hline high & 24 & 12400 & 3 \\
\hline
\end{tabular}

Table 6. Experiment classes 


\section{List of Figures}

- Figure 1. Average flow time with one manufacturing operation

- Figure 2. Tardiness with one manufacturing operation

- Figure 3. Average flow time with several manufacturing operations

- Figure 4. Throughput with several manufacturing operations

- Figure 5. Tardiness with several manufacturing operations

- Figure 6. Average flow time with inter-arrival fluctuation

- Figure 7. Tardiness with inter-arrival fluctuation

- Figure 8. Earliest Due Date Rule

- Figure 9. Tardiness - Earliest Due Date Rule 


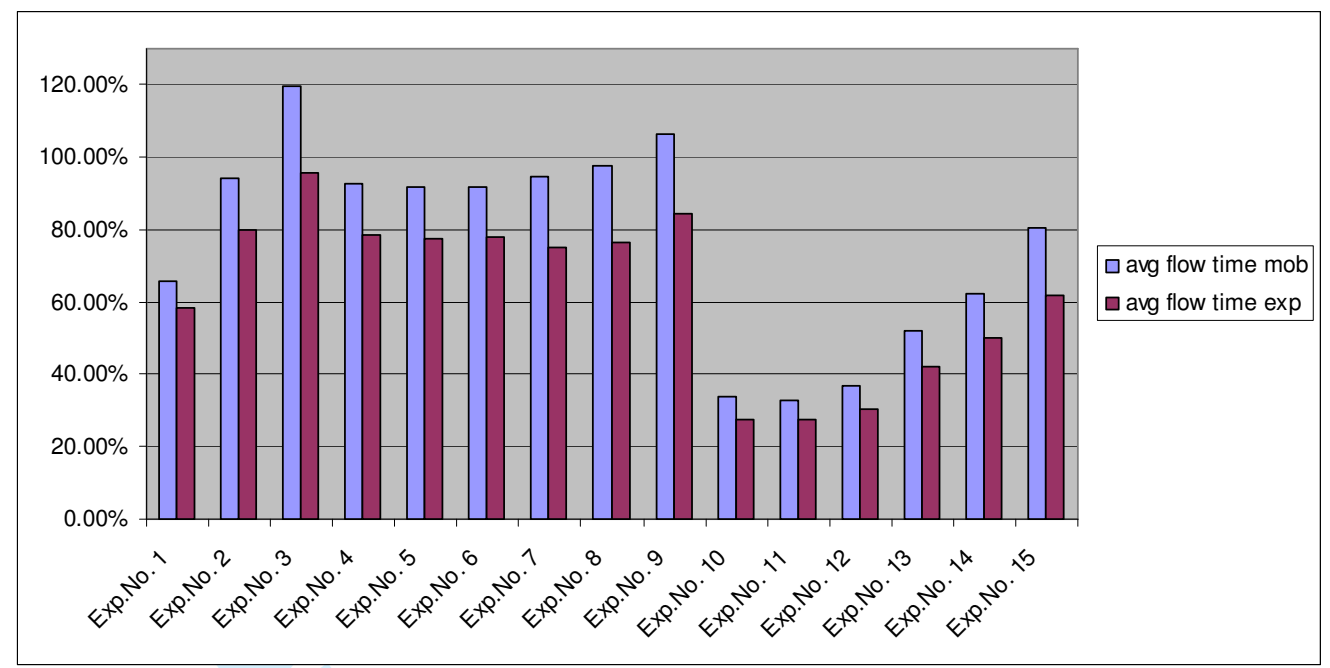

Figure 1. Average flow time with one manufacturing operation 


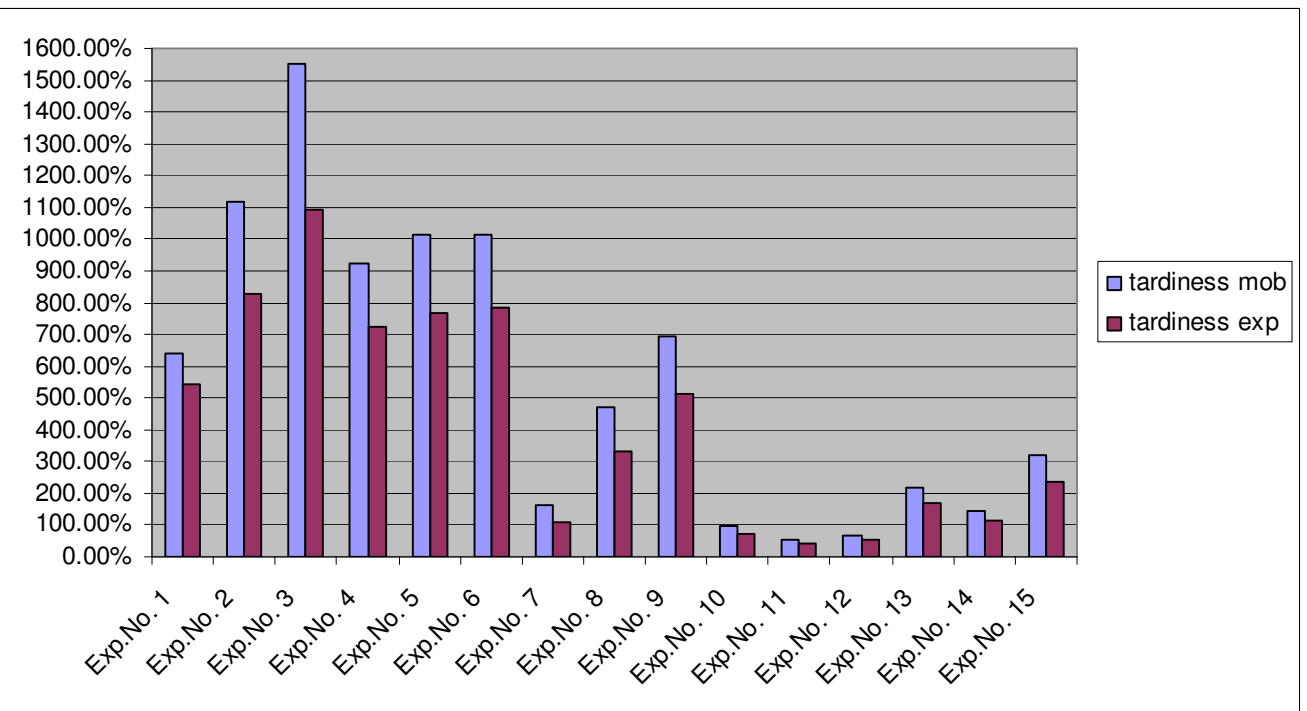

Figure 2. Tardiness with one manufacturing operation 


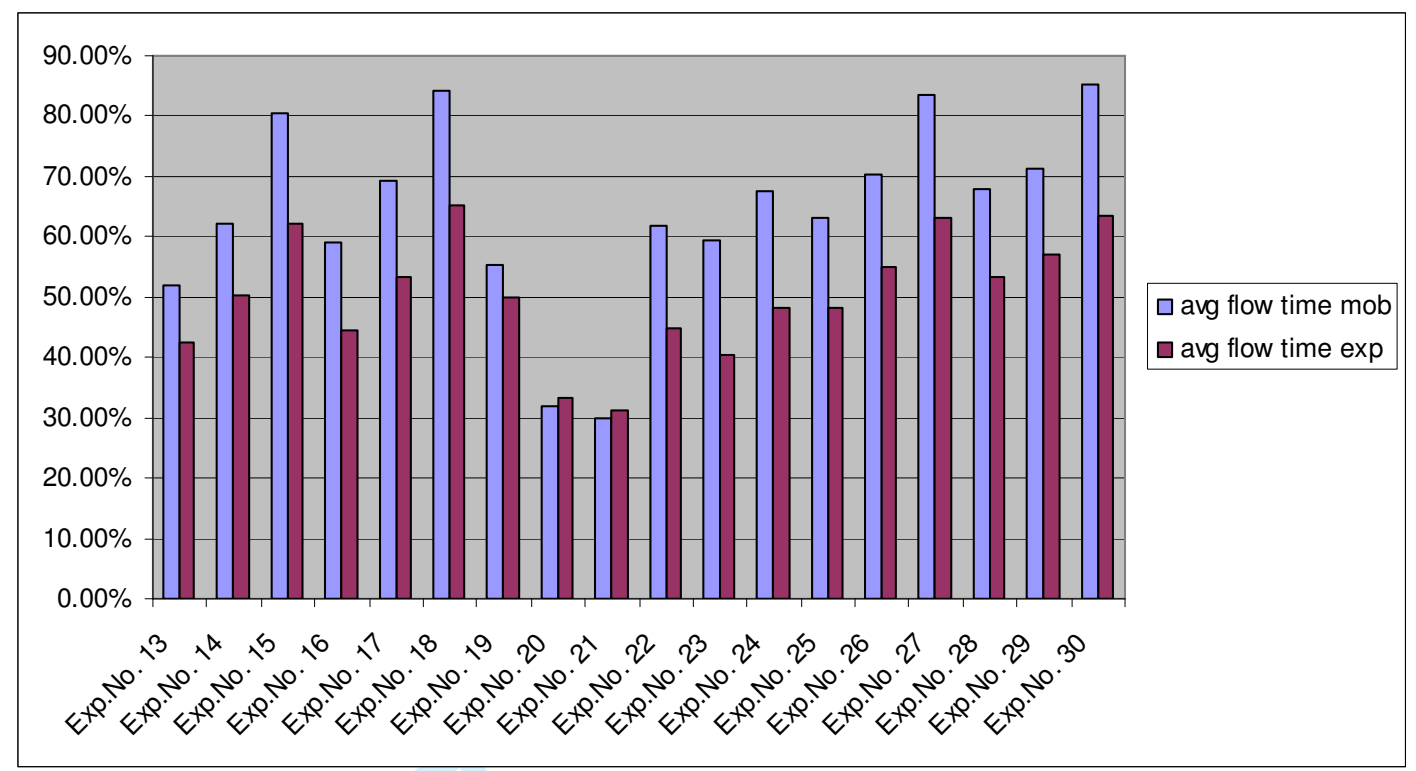

Figure 3. Average flow time with several manufacturing operations 


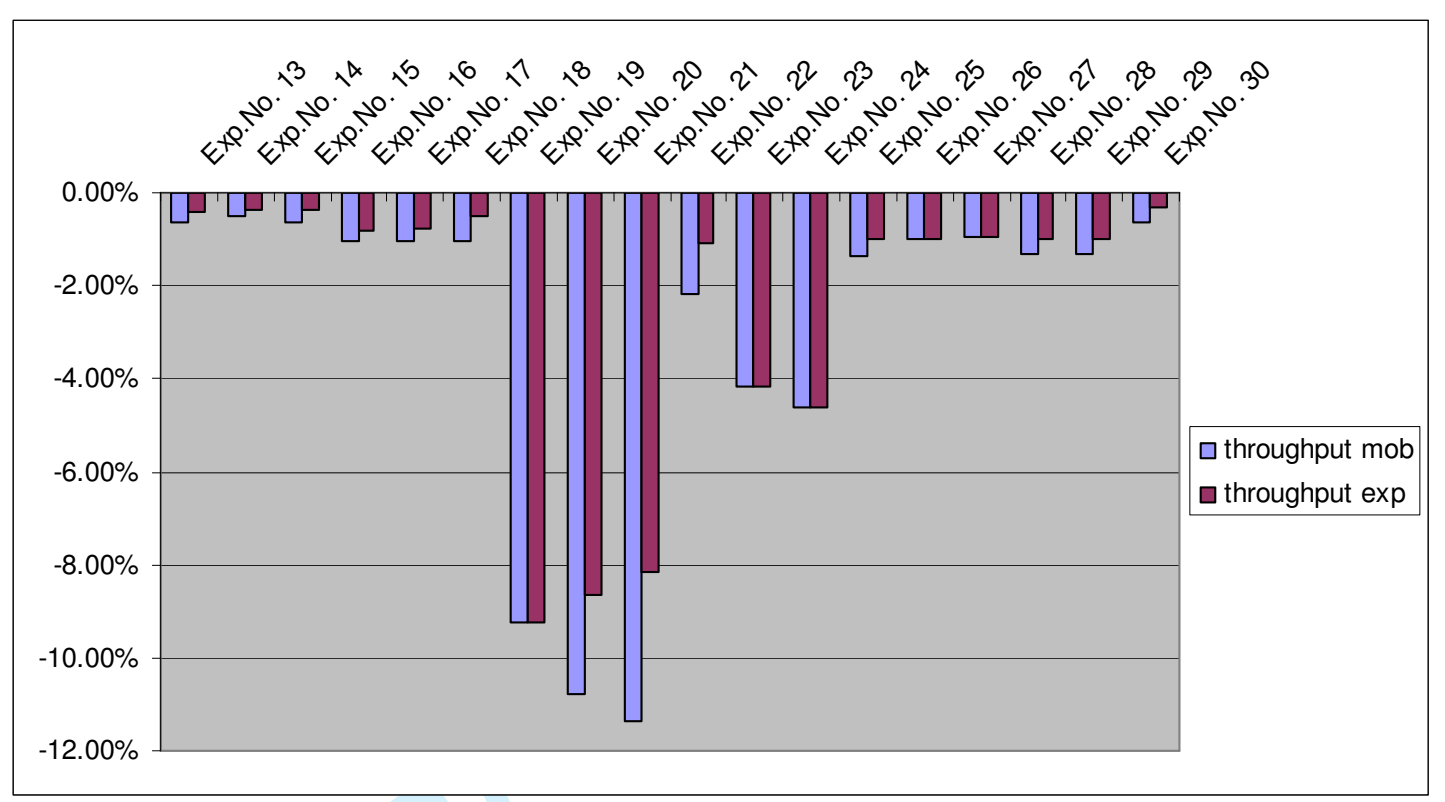

Figure 4. Throughput with several manufacturing operations 


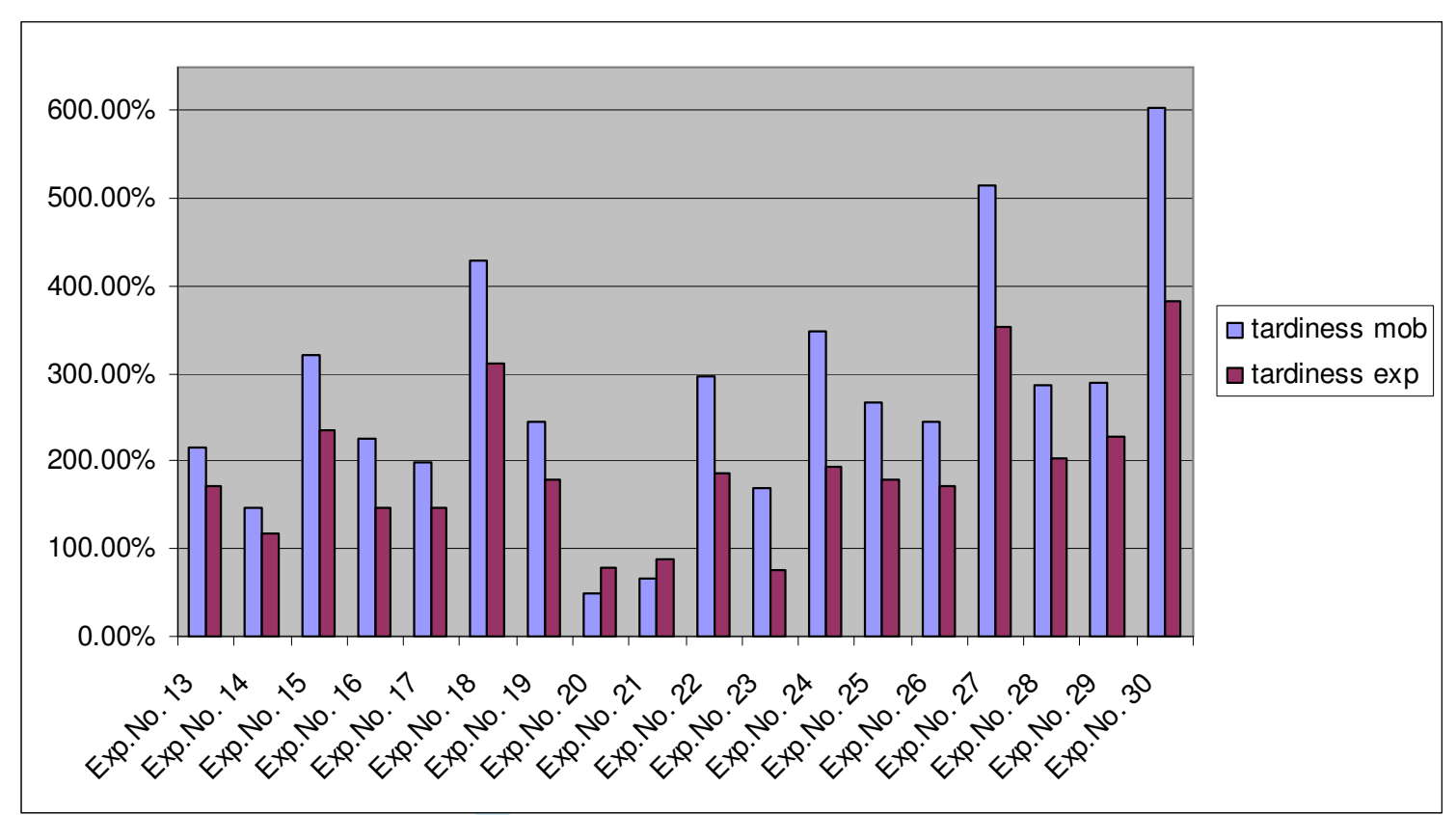

Figure 5. Tardiness with several manufacturing operations 


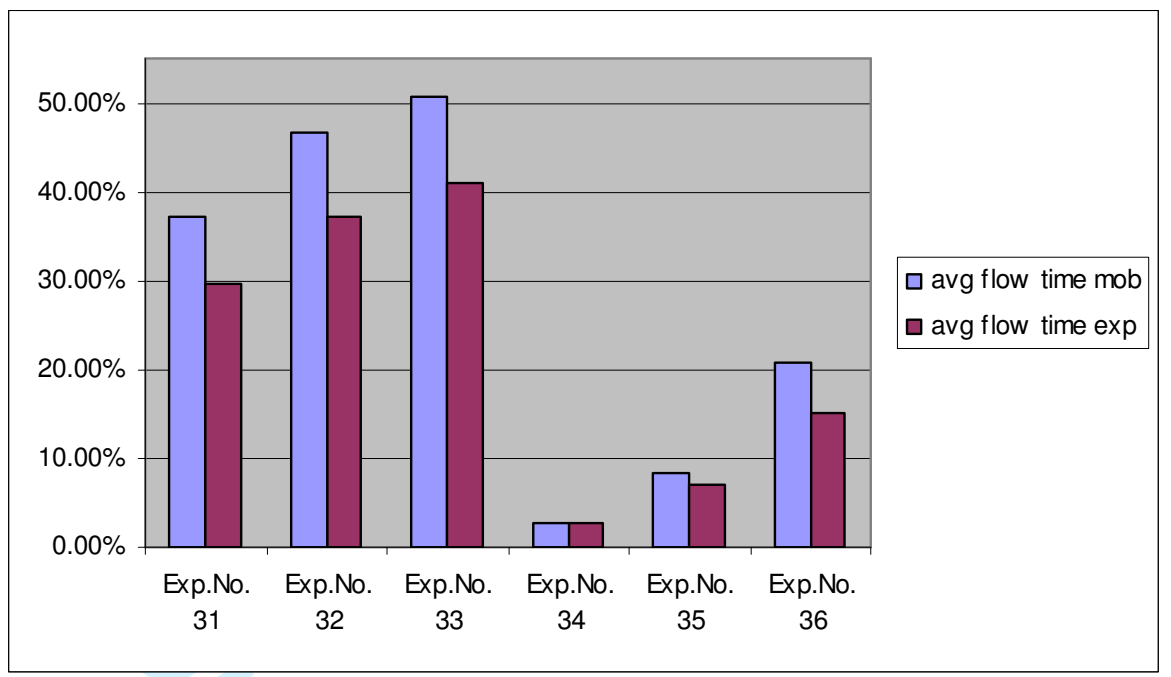

Figure 6. Average flow time with inter-arrival fluctuation 


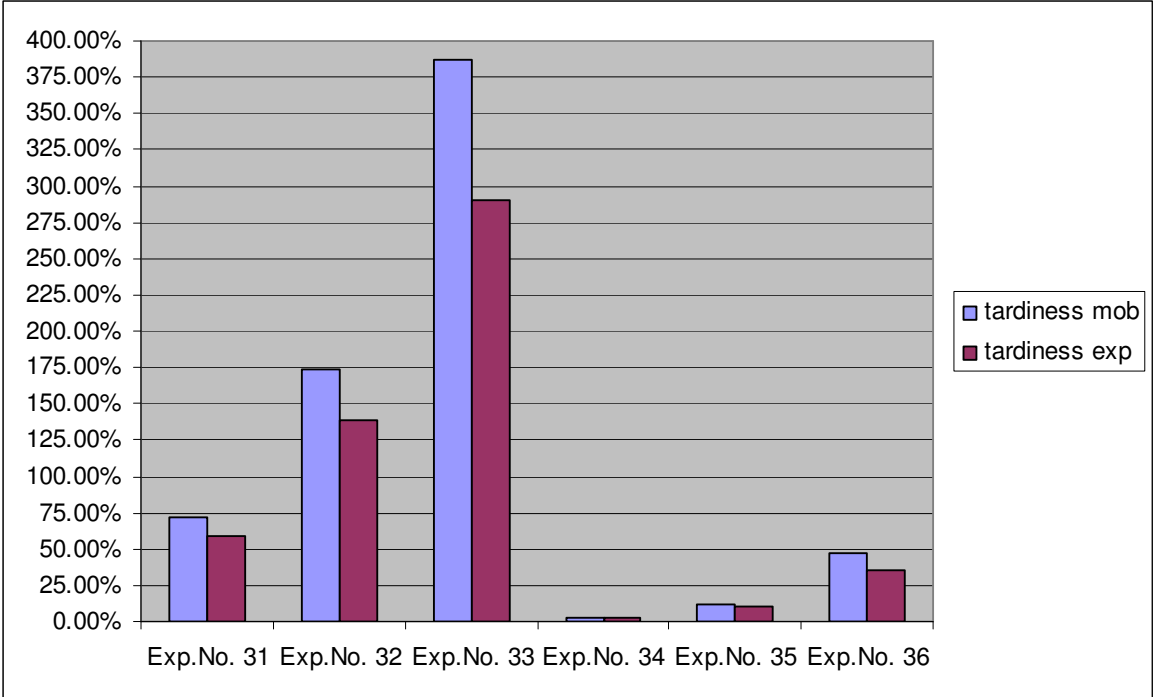

Figure 7. Tardiness with inter-arrival fluctuation 


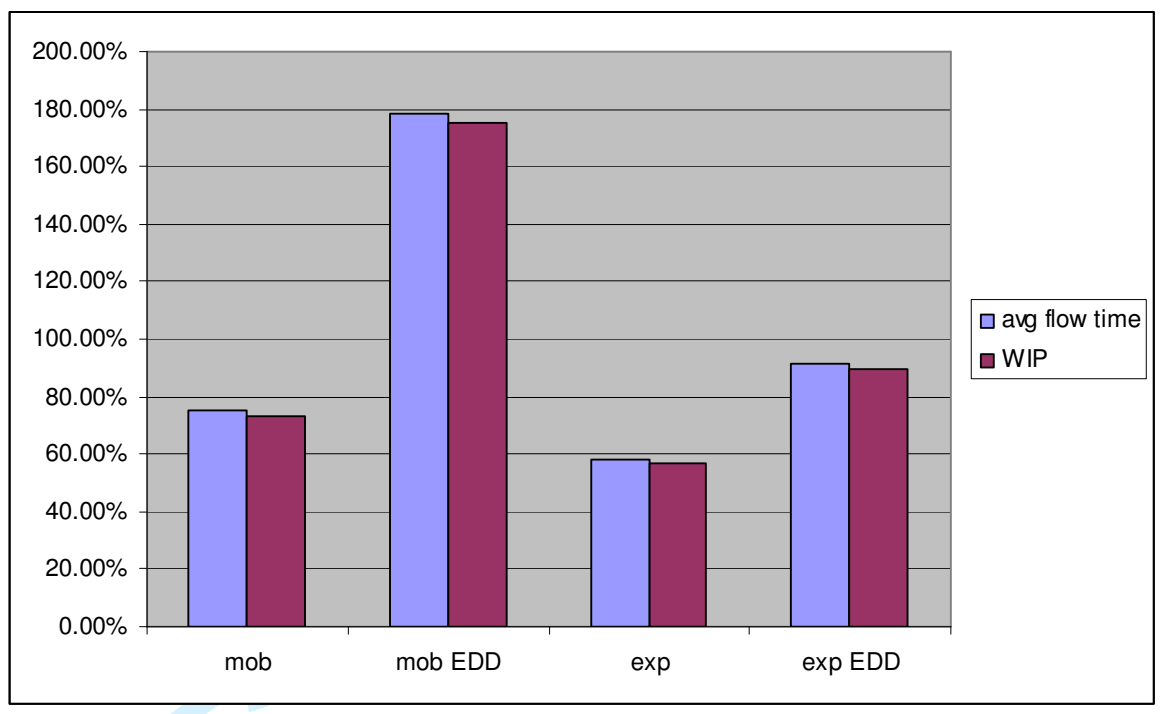

Figure 8. Earliest Due Date Rule 


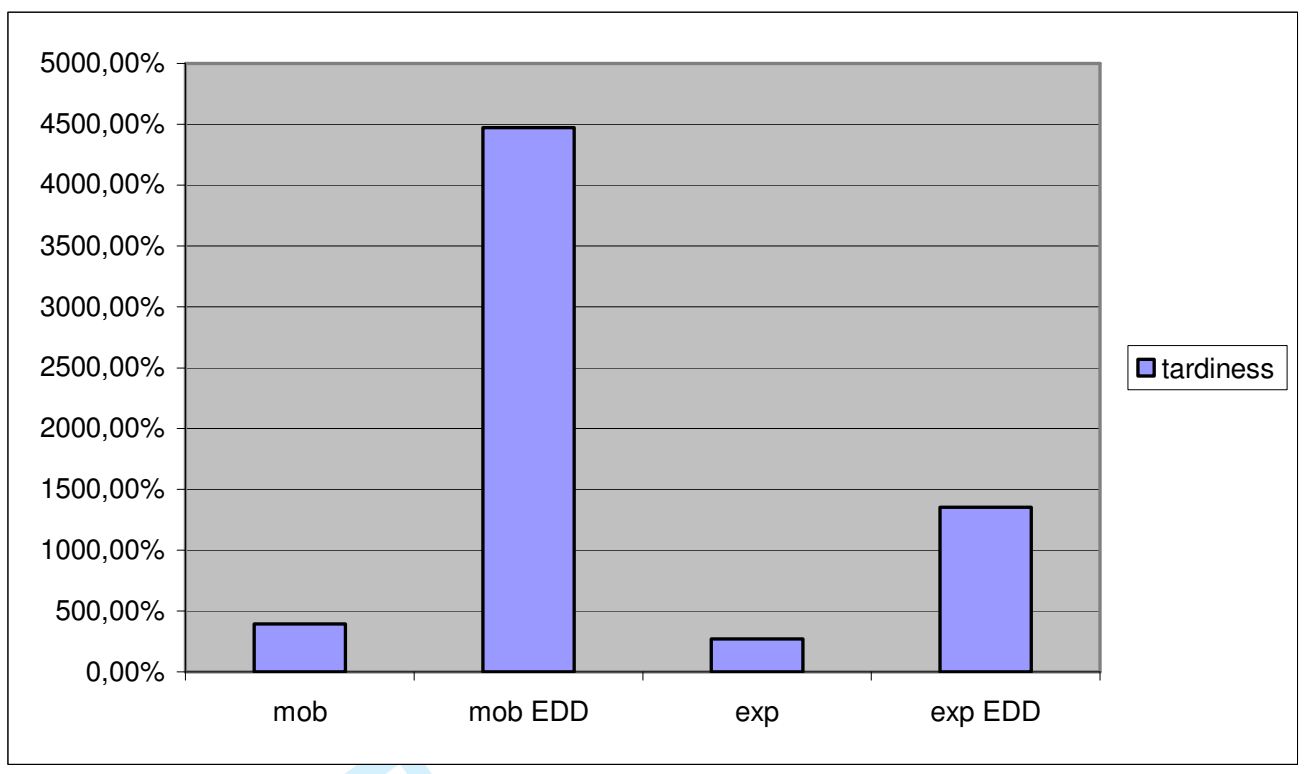

Figure 9.Tardiness - Earliest Due Date Rule 